Proceedings of the International Congress on Advances in Applied Physics and Materials Science, Antalya 2011

\title{
Effect of Dimple Potential on Ultraslow Light in a Bose-Einstein Condensate
}

\author{
D. TARHAN ${ }^{a, *}$ AND H. $\mathrm{UNCU}^{b}$ \\ ${ }^{a}$ Department of Physics, Harran University, Osmanbey Yerleşkesi, Şanlıurfa, 63300 Turkey \\ ${ }^{b}$ Department of Physics, Adnan Menderes University, Aydın, Turkey
}

\begin{abstract}
We investigate the propagation of ultraslow optical pulse in atomic Bose-Einstein condensate in a harmonic trap decorated with a dimple potential. The role of dimple potential on the group velocity and time delay is studied. Since we consider the interatomic scattering interactions, nonlinear Schrödinger equation or the Gross-Pitaevskii equation is used in order to get the density profile of the atomic system. We find large group delays of order $1 \mathrm{~ms}$ in an atomic Bose-Einstein condensate in a harmonic trap with a deep dimple potential.
\end{abstract}

PACS: 42.50.Gy, 67.85.Hj

\section{Introduction}

The impressive demonstration of ultraslow light propagation through an atomic Bose-Einstein condensate (BEC) [1], utilizing electromagnetically induced transparency (EIT) [2], has promised an appealing application as a quantum memory [3]. Beside the ultracold atoms, slow optical pulses have been observed in various media like hot rubidium vapor [4]. In BECs, ultraslow optical pulse is delayed by the order of few microseconds [1]. Nevertheless large group delay of light was observed in hot rubidium gases [4]. More recently, large controllable time delays for such broadband pulses have been proposed [5]. It has been theoretically and experimentally shown that this delay time can be increased by increasing the atomic density [6]. Moreover, dimple potential can be used for increasing the time delay since the density of Bose-Einstein condensate can be increased by means of the dimple potentials [7]. Phase space density can be enhanced by an arbitrary factor by using a small dimple at the equilibrium point of the harmonic trapping potential [8]. Recently, such potentials are also proposed for efficient loading and fast evaporative cooling to produce large BECs [9].

If the atom-atom interactions cannot be neglected, the structure of ground state of BEC is described by the Gross-Pitaevskii equation [10]. If the scattering length $a_{s}$ is much less than the mean interparticle spacing, GrossPitaevskii equation describes the zero-temperature properties of the non-uniform Bose gas.

In this paper, we investigate the one-dimensional propagation of ultraslow optical pulse in an atomic BoseEinstein condensate in a harmonic trap decorated with a dimple potential which is located at the center of harmonic potential. We study the role of the dimple potential on the group velocity and the time delay. We model the dimple potential by a Gaussian function which has a narrow width value. Our calculations show that it is

\footnotetext{
* corresponding author; e-mail: dtarhan@harran.edu.tr
}

possible to observe large group delays up to $1 \mathrm{~ms}$ for an optical pulse in an atomic Bose-Einstein condensate which is trapped in harmonic potential decorated with a deep dimple. The paper is organized as follows: first of all the calculation of density profile of an atomic BEC system is briefly reviewed. After then, propagation of ultraslow light under EIT system is discussed. We present our calculations and discuss the results in result section. Finally, we conclude in the last section.

\section{Density profile of a trapped Bose gas with dimple potential}

The density profile of a Bose-Einstein condensate in an external potential can be approximated very well by means of the Thomas-Fermi approximation (TFA). The density of ground state is given by the absolute square of the ground state wave function: $n(r)=\left|\Psi_{0}(r)\right|^{2}$. The TFA is often a good approximation to assume that the total ultracold atomic density is in fact constant during the weak light field propagation. On the issue of the dimple, the validity of TFA depends on the length scale of the dimple. If it is much larger than the healing length, then TFA with the trap+dimple potential would work fine. The healing length or coherence length of the BEC is given by $\xi=\left(1 /\left(8 \pi n a_{s}\right)\right)^{1 / 2}[11]$. Here $n$ is the density of the ultracold atomic medium and we can take it as the peak density $(n=\rho(0))$ of the atomic system. We consider the range of parameters in this work within the range of validity of TFA. One can approximate total density profile of ultracold atomic system for 1D (ground state density) by [11] $\rho(z)=[\mu-V(z)] / U_{0}$. Here $U_{0}=4 \pi \hbar^{2} a_{s} / m$ where $m$ is atomic mass and $a_{s}$ is the atomic $s$-wave scattering length. $\mu$ is the chemical potential and can be evaluated by using the TFA. The chemical potential is determined from $N=\int \mathrm{d} z \rho(z)$. We consider the dimple potential which is modelled by a Gaussian function. We represent one-dimensional harmonic potential with a dimple as

$$
V(z)=\frac{1}{2} m \omega_{z}^{2} z^{2}-V_{0} \mathrm{e}^{-\left(\frac{z}{\sqrt{2} l_{z}}\right)^{2}},
$$

where $\omega_{z}$ is the trap frequency of the harmonic trap in 
the $z$ direction, and $V_{0}>0$ shows the strength (depth) of the dimple trap $\left(V_{d}=-V_{0} \mathrm{e}^{-\left(\frac{z}{\sqrt{2} l_{z}}\right)^{2}}\right)$ located at $z=0$. It is possible to increase $V_{0}$ from 0 to $1500-2000 \hbar \omega_{z}$ [12]. We use large values for the width of Gaussian function $l_{z}\left(\right.$ in $\left.G(z)=-V_{0} \mathrm{e}^{-\left(\frac{z}{\sqrt{2} l_{z}}\right)^{2}}\right)$ compared to the extension of BEC (see Sect. 4) in order to get an appropriate modeling for the narrow dimple. If we apply a deep dimple to the atomic condensate in a harmonic trap, we will get the total number of atoms analytically by

$$
N=\int_{0}^{\sqrt{\frac{2\left(\mu+V_{0}\right)}{\left(m \omega_{z}^{2}+\frac{V_{0}}{l_{z}^{2}}\right)}}} \mathrm{d} z\left(\mu-\frac{1}{2} m \omega_{z}^{2}+V_{0} \mathrm{e}^{-\left(\frac{z}{\sqrt{2} l_{z}}\right)^{2}}\right) / U_{0} .
$$

By taking the integral in Eq. (2), we find an analytical expression for total number of atoms in terms of chemical potential, interaction term, trap frequency and strength of the dimple potential

$$
\begin{aligned}
N & =\frac{l_{z}}{3 \sqrt{2} U_{0}\left(V_{0}+m \omega_{z}^{2} l_{z}^{2}\right)^{\frac{3}{2}}}\left\{2 \sqrt{\mu+V_{0}}\right. \\
& \times\left[3 \mu V_{0}+m \omega_{z}^{2} l_{z}^{2}\left(2 \mu-V_{0}\right)\right]+3 V_{0}\left(V_{0}+m \omega_{z}^{2} l_{z}^{2}\right) \\
& \left.\times \sqrt{\left(V_{0}+m \omega_{z}^{2} l_{z}^{2}\right) \pi}\right\} \operatorname{erf}\left(\sqrt{\frac{\mu+V_{0}}{V_{0}+m \omega_{z}^{2} l_{z}^{2}}}\right) .
\end{aligned}
$$

Here $\operatorname{erf}(x)$ is the error function. The error function can be expanded in terms of $x$ where $x=\sqrt{\frac{\mu+V_{0}}{V_{0}+m \omega_{z}^{2} l_{z}^{2}}}$. $\operatorname{erf}(x)=\frac{1}{\sqrt{\pi}}\left[2 x-2 \frac{x^{3}}{3}+\frac{x^{5}}{5}-\frac{x^{7}}{21}+\mathrm{O}[x]^{9}\right]$. We insert this expanded term into Eq. (3) and solve this equation numerically in order to get chemical potential. Doing this we find the value of the chemical potential $\mu=1.0800 \times 10^{-12} \mathrm{eV}$ for $m=23 \mathrm{amu}, \omega=200 \mathrm{~Hz}$, and $V_{0}=100 \hbar \omega_{z}=6.582110^{-13} \mathrm{eV}$ for $N=1 \times 10^{6}$.

\section{Ultraslow light under EIT scheme}

We consider an EIT model for a gas of $N$ three-level atoms interacting with two laser beams in $\Lambda$ configuration. The upper level is coupled to the lower levels via a strong drive field with frequency $\omega_{\mathrm{c}}$ and a weak probe field of frequency $\omega_{\mathrm{p}}$. At resonance the absorption of the probe field can be neglected. Weak probe beam propagates along the condensate axis in the $z$ direction. Propagation of the ultraslow wave packet in one-dimensional inhomogeneous atomic condensate can be described by $[13,14]$ :

$$
\frac{\partial E}{\partial z}+\alpha(z) E+\frac{1}{v_{\mathrm{g}}(z)} \frac{\partial E}{\partial t}+\mathrm{i} b_{2}(z) \frac{\partial^{2} E}{\partial t^{2}}=0,
$$

where $\alpha(z)$ is the pulse attenuation factor; $v_{\mathrm{g}}(z)$ is the group velocity, and $b_{2}(z)$ is the group velocity dispersion. The third order dispersion is found to be much smaller and neglected [14]. EIT susceptibility [15] for the BoseEinstein condensate of atomic density $\rho$ is expressed as $\chi=\rho \chi_{1}$ with

$$
\chi_{1}=\frac{|\mu|^{2}}{\varepsilon_{0} \hbar} \frac{\mathrm{i}\left(\frac{\Gamma_{2}}{2}-\mathrm{i} \Delta\right)}{\left(\frac{\Gamma_{2}}{2}-\mathrm{i} \Delta\right)\left(\frac{\Gamma_{3}}{2}-\mathrm{i} \Delta\right)+\frac{\Omega_{\mathrm{c}}^{2}}{4}},
$$

where $\Delta=\omega-\omega_{0}$ is the detuning of the probe field frequency $\omega$ from the atomic resonance $\omega_{0}$. In Eq. (5), $\Omega_{\mathrm{c}}$ is the Rabi frequency of the control field; $\mu$ is the dipole matrix element for the probe transition. $\Gamma_{2}$ and $\Gamma_{3}$ denote the dephasing rates of the atomic levels. The significant position dependent group velocity for the optical pulse propagation can be calculated from the susceptibility using the relation [13]:

$$
\frac{1}{v_{\mathrm{g}}}=\frac{1}{c}-\left.\frac{\pi}{\lambda} \frac{\partial \chi}{\partial \omega}\right|_{\omega_{0}} .
$$

Here we take $\lambda=589 \mathrm{~nm}$ and $\omega_{31}=2 \pi c / \lambda$. In Eq. (6) group velocity depends on atomic density. As mentioned in the introduction, the atomic density can be increased by using a dimple potential [7]. An optical ultraslow pulse propagates through the ultracold medium without absorption due to the small imaginary part of the EIT susceptibility at resonance.

\section{Results and discussions}

We consider a gas of $N=1 \times 10^{6}$ of $\mathrm{Na}$ atoms with $\gamma=6 \mathrm{MHz}, \Gamma_{3}=0.5 \gamma, \Omega_{\mathrm{c}}=0.5 \gamma$, and $\Gamma_{2}=6 \times 10^{3} \mathrm{~Hz}$. We take accessible experimentally parameters such as $\omega_{\mathrm{r}}=$ $350 \mathrm{~Hz}, \omega_{z}=100 \mathrm{~Hz}$, so that peak density $\rho_{0}=1.56 \times 10^{20}$ $1 / \mathrm{m}^{3}$. Equation (3) is solved numerically in order to find the chemical potential. We assume that all atoms are loaded into the harmonic potential with a dimple, therefore effective length of the atomic medium becomes smaller to the order $6 \mu \mathrm{m}$. The density of the condensate is mainly controlled by the dimple potential for extremely deep dimple. We present the change of $\mu$ as a function of $V_{0}$ in Fig. 1. As the strength of the dimple potential increases, chemical potential becomes larger.

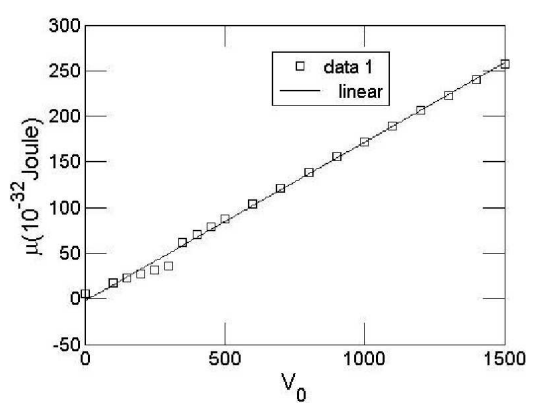

Fig. 1. The chemical potential $\mu$ vs. strength of dimple potential for ${ }^{23} \mathrm{Na}$ Bose-Einstein condensate of $N=$ $1 \times 10^{6}$ atoms. The squares show the chemical potential which correspond to the strength of the dimple potential. Solid line represents the linear fitting. Dimple potential $\left(V_{0}\right)$ is scaled by $\hbar \omega_{z}$. The parameters used are $M=23 \mathrm{amu}, a_{s}=2.75 \mathrm{~nm}$.

When light enters the condensate, its group speed exhibits a dramatic slowdown. Here we consider resonant 


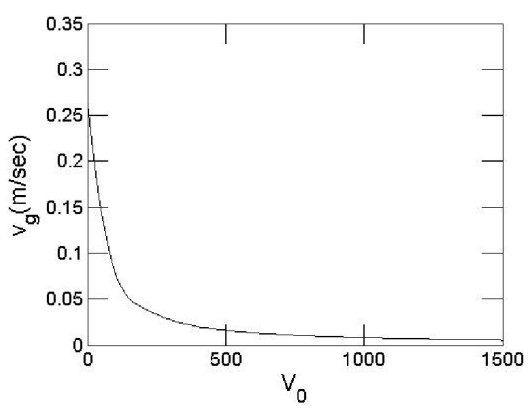

Fig. 2. Group velocity vs. strength of dimple potential for $\Delta=0$, propagating through a ${ }^{23} \mathrm{Na}$ Bose-Einstein condensate under EIT scheme for $N=1 \times 10^{6}$ atoms with $\gamma=6 \mathrm{MHz}, \Gamma_{3}=0.5 \gamma, \Omega_{\mathrm{c}}=0.5 \gamma$, and $\Gamma_{2}=6 \times 10^{3} \mathrm{~Hz}$. The solid line shows the peak value of the group velocity vs. strength of the dimple potential. Dimple potential $\left(V_{0}\right)$ is scaled by $\hbar \omega_{z}$.

probe pulse with $\Delta=0$. Within the condensate region, at zero temperatures, the group velocity remains approximately at the same ultraslow value. Light rapidly accelerates to high speeds when it leaves the condensate at the interface to thermal part. At extremely low temperatures, $\rho_{0}$ saturates to the Thomas-Fermi density. At zero temperature, $v_{\mathrm{g}_{0}}$ can be calculated by $\rho_{0}$ which was converted from the Thomas-Fermi approximation. We can ignore the spatial variations therefore density of the atomic system can be taken as $\rho(z=0)=\rho_{0}$. In other words, at low temperatures group velocity is determined by $\rho_{0}$ and group velocity decreases rapidly with the increasing strength of the dimple for small dimple strengths as seen in Fig. 2. However, group velocity decreases slowly at deep dimple. One can interpret the decrease in the rate of slow down as follows: as $V_{0}$ becomes larger, the dimple part predominates over the harmonic part of the potential.

The group velocity is almost constant within the ultracold atomic medium. In this case we ignore the small contributions of modal and waveguide dispersions and determine the group velocity, the same for both fractions, by assuming a constant peak density of the condensate in the material dispersion relation. So the delay time can be calculated approximately by the time delay formula $t_{D}=L_{z} / v_{g}$. Here $t_{D}$ is the time delay of the ultraslow pulse, $v_{g}$ is the group velocity in the z direction and $L_{z}$ is the axial length of the condensate which can be taken as: $L_{z}=2 R . R$ is the Thomas-Fermi axial radius which is given as $\sqrt{2\left(\mu+V_{0}\right) /\left(m \omega_{z}^{2}\right)} . \quad v_{g}$ is calculated by using Eq. (6). Therefore we find large group delays of order $1 \mathrm{~ms}$ in an atomic Bose - Einstein condensate in a harmonic trap with a deep dimple potential in which the strength of the deep dimple potential is $V_{0}=1500 \hbar \omega_{z}$.

\section{Conclusion}

We have explored the propagation of ultraslow light through a Bose-Einstein condensate in a harmonic trap with a dimple potential. We have investigated the effect of the dimple potential on the group velocity and the time delay. As the strength of the dimple potential increases, group velocity becomes smaller. However, at a critical value of the dimple group velocity becomes approximately constant. As a result, time delay can be increased by means of an atomic Bose-Einstein condensate in a harmonic trap with a dimple potential. In other words, large group delay can be obtained by using dimple potential in ultracold atomic system.

\section{Acknowledgments}

H.U. acknowledges support by TUBITAK TBAG (108 T003). D.T. was supported by TUBITAK-Kariyer grant No. 109 T686.

\section{References}

[1] L.V. Hau, S.E. Harris, Z. Dutton, C.H. Behroozi, Nature 397, 594 (1999).

[2] S.E. Harris, Physics Today 50, 36 (1997).

[3] C. Liu, Z. Dutton, C.H. Behroozi, L.V. Hau, Nature 409, 490 (2001).

[4] M.M. Kash, V.A. Sautenkov, A.S. Zibrov, L. Hollberg, G.R. Welch, M.D. Lukin, Y. Rostovtsev, E.S. Fry, M.O. Scully, Phys. Rev. Lett. 82, 5229 (1999).

[5] Q. Sun, Y.V. Rostovtsev, J.P. Dowling, M.O. Scully, M.S. Zubairy, Phys. Rev. A 72, 031802(R) (2005).

[6] M. Klein, Y. Xiao, A.V. Corshkov, M. Hohensee, C.D. Leunga, M.R. Browning, D.F. Phillips, I. Novikova, R.L. Walsworth, Proc. SPIE 6904, 69040C (2008).

[7] P.W. Pinkse, A. Mosk, M. Weidemüller, M.W. Reynolds, T.W. Hijmans, J.T.M. Walraven, Phys. Rev. Lett. 78, 990 (1997).

[8] D.M. Stamper-Kurn, H.-J. Miesner, A.P. Chikkatur, S. Inouye, J. Stenger, W. Ketterle, Phys. Rev. Lett. 81, 2194 (1998).

[9] D. Comparat, A. Fioretti, G. Stern, E. Dimova, B.L. Tolra, P. Pillet, Phys. Rev. A 73, 043410 (2006).

[10] L.P. Pitaevskii, Zh. Eksp. Teor. Fiz. 40, 646 (1961); E.P. Gross, Nuovo Cimento 20, 454 (1961).

[11] C.J. Pethick, H. Smith, Bose-Einstein Condensation in Dilute Gases, Cambridge University Press, Cambridge 2002.

[12] N.P. Proukakis, J. Schmiedmayer, H.T.C. Stoof, Phys. Rev. A 73, 053603 (2006).

[13] S.E. Harris, J.E. Field, A. Kasapi, Phys. Rev. A 46, R29 (1992).

[14] D. Tarhan, A. Sennaroglu, Ö. Müstecaplıõ̃lu, J. Opt. Soc. Am. B 23, 1925 (2006).

[15] M.O. Scully, M.S. Zubairy, Quantum Optics, Cambridge Univ. Press, Cambridge 1997. 